\title{
La ética decolonial y su relación con el Estado de derecho en cuanto a la interculturalidad y la plurinacionalidad en el Ecuador
}

Fecha de recepción : 04 de julio de 2019 • Fecha de aceptación: 5 de septiembre de 2019 • Fecha de publicación: 10 de septiembre de 2019

\author{
Dra. Mireya Katerine Pazmiño Arregui \\ Universidad Estatal de Bolívar \\ mkpazmino@ueb.edu.ec3 \\ https://orcid.org/0000-0002-5352-7303 \\ Dr. Gonzalo Enrique Noboa Larrea \\ Universidad Estatal de Bolívar \\ gnoboa@ueb.edu.ec \\ https://orcid.org/0000-0001-5690-7144 \\ Dr. Telmo Elías Yánez Olalla \\ Universidad Estatal de Bolívar \\ tyanez@ueb.edu.ec \\ https://orcid.org/0000-0003-2588-9101
}

\section{Resumen}

El objetivo de la investigación es determinar la perspectiva existente de la ética decolonial y lo que significa para el Estado ecuatoriano la interculturalidad y la plurinacionalidad instauradas constitucionalmente. La ética decolonial es una herramienta de transgresión que se relaciona con los procesos internos de la globalización. Resulta oportuno resaltar que la Constitución de la República del Ecuador expresa claramente que Ecuador es un Estado de derecho y justicia social, democrático, soberano, independiente, unitario, intercultural, plurinacional y laico, el cual es irrenunciable e imprescriptible. El estudio es de tipo cualitativo-descriptivo, apoyado en la hermenéutica y el modelo de la comparación. El propósito del estudio es profundizar acerca del significado de la ética decolonial y compararla con lo que persigue el Estado ecuatoriano en su Constitución, y al mismo tiempo cuestionar el conocimiento de la ética decolonial hacia el proceso pluriculturalista que ofrece el Estado ecua- 
toriano con la intención de regir una serie de principios éticos que proporcionan respecto, seguridad e igualdad entre los habitantes. Finalmente, este estudio quiere aprovechar que constitucionalmente el Estado promueve y respeta la plurinacionalidad, para desarrollar un plan integrador adaptado al entorno y al derecho de los ciudadanos del Ecuador.

Palabras clave: estado de derecho, ética decolonial, interculturalidad, integración, plurinacionalidad, seguridad.

\begin{abstract}
The objective of the research is to determine the existing perspective of decolonial ethics, and what it means for the Ecuadorian State interculturality and plurinationality constitutionally established. The decolonial ethic is a tool of transgression that is related to the internal processes of globalization. It should be noted that the Constitution of the Republic of Ecuador clearly states that it is a State of rights and justice, social, democratic, sovereign, independent, unitary, intercultural, plurinational and secular which is inalienable and imprescriptible. The study is qualitative-descriptive based on the hermeneutics and the comparison model. The purpose of the study is to deepen the meaning of decoIonial ethics and compare it with what the Ecuadorian State pursues in its Constitution. Question the knowledge of decolonial ethics towards the pluriculturalist process offered by the Ecuadorian State, with the intention of governing a series of ethical principles that provide respect, security and equality among the inhabitants. Finally, this study wants to take advantage of the fact that the State constitutionally promotes and respects plurinationality, to develop an integrative plan adapted to the environment and the law of the citizens of Ecuador.
\end{abstract}

Keywords: rule of law, decolonial ethics, interculturality, integration, plurinationality, security. 


\section{Introducción}

Los derechos humanos constituyen una de las singulares contradicciones del mundo actual (Vitória, 2017). Sin embargo, como destaca Villoro (2000), los derechos contribuyen a respaldar los procesos de lucha contra la explotación, la dominación de las razas y la descalificación de los seres humanos por otras personas que se creen superiores, fomentando sus esperanzas en un mundo más igualitario, justo y pluricultural.

En ese mismo sentido, Shiva (citado en Acosta y Martínez, 2011) expresa que, cuando no existe un respeto hacia los derechos humanos, la ética y los Estados plurinacionales e interculturales, se empiezan a desatar una serie de acontecimientos que atentan contra la igualdad, la democracia, las razas, la cultura y los idiomas, dando inicio a agresiones y desesperanza social.

La investigación se basa en conocer acerca de la ética decolonial, a la cual Maldonado-Torres (2011) define como una relación política, social y económica, donde se destaca la soberanía de los pueblos, en conjunto con el poder que se les otorga a sus pobladores para construir una nación. También el estudio destaca la importancia que el estado ecuatoriano les otorga por vía constitucional a sus pobladores respecto a la interculturalidad y la plurinacionalidad.

Como expresa Almeida (2018), cuando se habla de la interculturalidad en el Ecuador hay que señalar que, aunque en el país existe una diversidad significativa tanto en el orden cultural como étnico, no ha sido del todo reconocida por el Estado, llegando incluso muchos de sus exponentes a ser apartados del sistema social. Almeida (2018) apunta que realmente no se les ha otorgado nunca el sitio que merecen estos grupos indígenas y afroecuatorianos, desaprovechando sus valores, idiomas y sobre todo su reconocimiento ancestral. Respecto a la plurinacionalidad, es importante lo que atestigua nuestra historia: ha sido ocultada sin que se realizara un esfuerzo serio por conocer a los pueblos indígenas y afroecuatorianos, por apreciar sus valores y entender sus especificidades. En realidad, ni siquiera la mayoría mestiza ha hecho un esfuerzo por entenderse a sí misma.

A todas estas, es necesario comenzar a identificar las características de la plurinacionalidad del Estado ecuatoriano en el ámbito constitucional. En este sentido, en el artículo N.$^{\circ} 1$ de la Constitución de la República del Ecuador (2008), se reconoce lo plurinacional desde el punto de vista de los derechos y la justicia social, democrática, soberana e independiente, dándole un rango de irrenunciabilidad e imprescriptibilidad. Bajo esta perspectiva, este reconocimiento estatal no se divorcia de los parámetros de investigación, que, por medio de las distintas metodologías epistemológicas, busca rescatar la ética desde la perspectiva decolonizadora, aprovechando el apoyo o soporte constitucional.

La investigación se apoya en la metodología de la comparación (Pliscoff y Monje, 2003), la cual se adapta a todo tipo de enseñanza y permite ordenar estratégicamente las vertientes de la ética decolonial, la interculturalidad y el plurinacionalismo. Lo resaltante en este método es que permite el entendimiento sistemático de similitudes hermenéuticas con la finalidad de estudiar sus semejanzas positivas o negativas para que finalmente se planteen conclusiones y discusiones que se adapten y orienten a la resolución del problema de investigación. 
Para finalizar, la investigación se direcciona hacia la comprensión de lo que significa la ética decolonial y su relación con el Estado de derecho en cuanto a la interculturalidad y la plurinacionalidad en el Ecuador con la intención de conocer lo que establecen las Leyes y lo que sucede en la realidad, a fin de proponer salidas positivas para realzar la cultura en la población ecuatoriana.

\section{La ética decolonial y sus implicaciones axiológicas}

La ética, desde el punto de vista de la investigación, no puede tener otra dirección que mantener en primera línea los principios de justicia y de solidaridad humana. En este sentido, Walzer (2001) pretende realzar estos principios dándole intensidad a esos valores que, de alguna manera, se esparcen de manera sustantiva con una doble intencionalidad entre grupos de personas que buscan un beneficio propio, sin pensar en lo más mínimo en el colectivo.

Con referencia a lo anterior, Kant (1996) expresó que la ética, desde el punto de vista de la concepción pragmática de la educación y de la formación, se inclina hacia la importancia de crear elementos morales, éticos y sociales por parte de los actores del entorno educativo, el cual permite accionar valores necesarios tales como la racionalidad y la rectitud moral de las acciones humanas. Kant dividía la ética en dos grandes grupos: el imperativo categórico y el hipotético. En ambos casos se hace presente la moral y las buenas costumbres, sin embargo, la gran diferencia, y es lo más destacable y relevante en la investigación, es que el imperativo hipotético estaba supeditado a Normas y Leyes que penaban a los que la infringían. Por lo tanto, se afirma que una moral sin ética puede ser considerada una abstracción vacía, pero una ética sin moral es totalmente oscura, ciega y peligrosa.

Ahora bien, al momento de conceptualizar el colonialismo o sistema decolonial, inmediatamente la referencia va direccionada hacia el dominio que unos pueblos ejercen sobre otros (Polo, 2016). Viéndolo desde esta perspectiva, la violencia y la tiranía se hacen presentes, creando ciertos círculos sociales que solo buscan beneficio propio sin importar cualquier transgresión o impacto psicosocial que la misma pueda conllevar a la población a la disgregación. El colonialismo, según Taylor (1993), tiene la tendencia de trasgredir culturas, pensamientos y derechos, una tendencia que ya no se reduce solamente a la parte política, sino que también afecta lo social, humano y psicológico, y atenta contra la libertad de expresión de pensamiento, de crecimiento y desarrollo intelectual, en pro de una civilización moderna y globalizada.

Por todo lo anterior, se afirma que la ética decolonial surge de propuestas filosóficas que cubren en cierta forma tres grandes contextos. El primer lugar, tenemos las teorías del reconocimiento; en segundo lugar, la filosofía intercultural, y finalmente las teorías decoloniales (Céspedes, 2012). Cada una de ellas tiene su anclaje filosófico en el resguardo de desarrollar y respetar los valores que representan y benefician la vida humana en todos sus aspectos morales, interculturales y sociales.

En el contexto de la ética decolonial, se aborda especialmente el reconocimiento de los derechos de los seres humanos y la interculturalidad (Fonseca y Jerrems, 2012). Por intermedio de la ética 
decolonial se da un sentido transversal para reconocer las implicaciones morales que esta trae consigo; es decir, se realza el sentido de pertenencia en todos los aspectos culturales, sociales, políticos que de alguna manera permita el desarrollo de los pueblos y el enriquecimiento de las posturas en cuanto a legitimidad, que, en este caso, les pudieran concebir por intermedio de Leyes y Decretos para que se cumpla en igualdad de condiciones.

La ética decolonial debe hacerse sentir por todos aquellos actores que desean que la igualdad de condiciones sea la vanguardia para el éxito de la sociedad (Walsh, 2013). Por ejemplo, en Ecuador tomando en consideración el contexto de la globalización como parte de la modernización del Estado, es importante la incursión de sus etnias, no solo desde el punto de vista de la cultura y de la artesanía, también es necesaria su incursión en el mundo de la educación superior, con la intención de intensificar y ramificar una serie de atributos y de acceder de manera eficiente y acorde a la modernidad y la tecnología a los nuevos tiempos.

La ética decolonial se convierte en el proceso por el cual se libera la autonomía y hace un llamamiento a una pedagogía para reconstruir una nueva humanidad (Soazo, 2017). Una sociedad netamente pensante, aprovecha todas las ventajas pedagógicas -y la significancia de la decolonialidad - que permitan activar conocimientos actualizados y competitivos que se adicionen a la lucha social y moral para alinear pensamientos positivos en busca de adherirse a la globalización.

Los seres humanos y su conocimiento deben estar descolonizados, lo que quiere decir que deben estar preparados para instaurar y constituir una sociedad pensante y cuestionadora.

\section{Estado plurinacional en Ecuador y su Estado de derecho}

El plurinacionalismo, o la plurinacionalidad, se refiere a la convivencia correlacional de dos o más grupos nacionales dentro de un mismo Estado o Gobierno (Paz, 2015). Sigue expresando Paz que, en Sudamérica, existen dos países que asumen el plurinacionalismo como parte de sus derechos y deberes constitucionales, estos países son Bolivia y Ecuador. Inclusive el segundo lo soporta en la Constitución, en el artículo N. ${ }^{\circ}$, donde se declara que Ecuador es un Estado constitucional de derechos y justicia, social, democrático, soberano, independiente, unitario, intercultural, plurinacional y laico. Se organiza en forma de República y se gobierna de manera descentralizada (Constitución de la República del Ecuador, 2008).

La población del Ecuador es constitucionalmente plurinacional, en solidaridad y estructura democrática, por lo tanto, es un deber del Estado que se acate y se cumpla el mencionado artículo N. ${ }^{\circ}$ 1. Cabe destacar el comentario de Acosta (2010) donde expresa que el reconocimiento del Estado plurinacional ecuatoriano resulta un avance significativo, pero será insuficiente si no existe un empuje y respaldo por parte de todos los involucrados para construirlo de manera efectiva.

En consecuencia, no existe una relación directa o algo que trate de vincular directamente a los pobladores a integrarse; sin embargo, en el artículo N. ${ }^{\circ} 6$ de la Carta Magna ecuatoriana, se manifiesta que la nacionalidad se encuentra vinculada al marco jurídico político de las personas y su relación con el Estado, esto sin hacer prejuicio de su pertenencia a alguna de 
las nacionalidades étnicas que conviven en el Ecuador plurinacional. Unido a esto, dentro de la organización político administrativa que busca el Estado, se conforman circunscripciones territoriales étnicas que tienen el derecho y las competencias que le otorga constitucionalmente el Estado para tomar la descentralización en cuanto a sus principios de interculturalidad, plurinacionalidad, todo enmarcado dentro del marco legal de acuerdo con los derechos colectivos (artículo N. 257 de la Constitución de la República del Ecuador, 2008).

Por otra parte, el artículo $\mathrm{N} .^{\circ} 380$ de la Constitución ecuatoriana expresa que es responsabilidad del Estado velar, proteger, conservar, identificar, el crecimiento y difusión del patrimonio cultural tanto tangible como intangible, así como también de toda la riqueza histórica, artística, lingüística y artesanal, con la única intención de salvaguardar la identidad plurinacional, pluricultural y multiétnica del Ecuador.

Los derechos internacionales quedan totalmente respaldados por la Constitución del Ecuador, inclusive respetando la Declaración de las Naciones Unidas sobre los Derechos de los Pueblos Indígenas. Sin embargo, es bueno aclarar que, aunque este derecho no proporciona ninguna seguridad jurídica sobre la plurinacionalidad, sí deja claro que toda persona indígena tiene derecho a una nacionalidad. Los pueblos y las personas indígenas tienen derecho a pertenecer a una comunidad o nación indígena.

\section{La interculturalidad ecuatoriana y su importancia igualitaria}

Hablar de lo intercultural, descuidando la pluralidad, sería apartar realidades culturales que son bases sólidas para lograr la convivencia y el compartir entre etnias. Salazar (2016) define el término de la interculturalidad como un canal comunicativo para relacionar las culturas de una región. También la Convención sobre la Protección y Promoción de la Diversidad de las Expresiones Culturales de la UNESCO (2005) expresa que la interculturalidad es la presencia interactiva que trata a las culturas de manera equitativa para lograr la unificación de criterios étnicos permitiendo el dialogo, el respeto y la igualdad de condiciones dentro del Estado.

La difusión de contenidos interculturales es esencial para el reconocimiento de la población. Alavez (2014) se refiere a la interculturalidad como las relaciones de intercambio y comunicación igualitarias entre grupos culturales de diferentes costumbres étnicas, religión, lengua o nacionalidad.

Por todo lo anterior, la importancia que reviste la interculturalidad es basada en la construcción de una sociedad más democrática e igualitaria, aprovechando los procesos de interrelación y comunicación de saberes, así como de códigos, patrones y valores, entre los diferentes grupos culturales étnicos, entendiendo que existe igualdad entre sujetos, independientemente de la posición que ocupen en el sistema.

Ahora bien, la interculturalidad en Ecuador es conocida gracias a la diversidad en cuanto a sus pobladores étnicos, que de alguna manera definen o tienen bien delineadas su cultura y sus costumbres (Fernández, 2012). Sin embargo, es notorio que dicha situación no es reconocida del todo por el Estado y, aunque se encuentre visiblemente notoria su existencia, no se le ha dado el 
debido valor de reconocimiento, haciendo contraste con la realidad escrita. En otras palabras, lo que está escrito no concuerda con lo que está sucediendo.

Expresa este autor (Fernández, 2012), que no es desconocido que, a lo largo de la historia ecuatoriana, el problema de la interculturalidad ha sido ocultada por el Estado, a pesar de los esfuerzos que han hechos tanto los pueblos indígenas como los afroamericanos para que aprecien sus valores, tratando de incursionar ante la sociedad como lo estipula la Constitución.

Si bien es cierto que se han producidos esfuerzos enormes para ir contrarrestando esta situación, también es cierto que el estado debe ejercer o actuar de forma coherente ante los discursos que se relacionan con el caso. Lo importante es tratar de constituir elementos centrales de desarrollo para el fortalecimiento de los vínculos interculturales de la región, para promover de manera coherente la justicia social y la diversidad cultural (Cuji, 2012).

No se trata simplemente de aceptar la realidad multiétnica o multicultural, se trata más bien de solidificar las bases necesarias que permitan exaltar la importancia de la interculturalidad con la finalidad de controlar la heterogeneidad ecuatoriana, y que, de una vez, permitan la actualización y la equidad de los grupos étnicos ante la sociedad.

La complejidad se hace presente durante este proceso intercultural, sin embargo, Arce (2017) reseña que la interculturalidad no es característica natural de todas las sociedades complejas, sino el objetivo final al que se debe llegar para lograr articular todo el proceso de forma coherente. El esfuerzo debe ser mancomunado, de forma tal que se puedan lograr avances significativos en esta materia.

La interculturalidad ecuatoriana no se debe quedar solamente en discursos anuales o en conversaciones poco profundas, deben sostenerse políticas concretas que ayuden a propiciar la participación y la inclusión de la población indígena de manera progresiva.

La idea de toda esta concentración de esfuerzos no es más que la búsqueda de superación de los prejuicios, el racismo, las desigualdades y las asimetrías que caracterizan actualmente al país en materia intercultural (Núñez y González, 2014). Se deben rescatar de manera contundente las condiciones de respeto, igualdad y desarrollo de espacios comunes.

Algo interesante que destacar es que, gracias a las condiciones expresadas anteriormente, se mantiene viva esa exclusión social, por lo tanto, un avance interesante para lograr el camino deseado para realzar la interculturalidad, es en primer lugar reconocer esas condiciones, para darle paso a las virtudes, habilidades y competencias que ayudarían de manera efectiva a consolidar el proceso de inclusión cultural.

Finalmente, a este punto, una sociedad intercultural es la que mantiene un proceso dinámico, sostenido y permanente de relación, comunicación y aprendizaje mutuo de costumbres y culturas étnicas con la población en general (Tirzo y Hernández, 2010). Allí se da un esfuerzo colectivo y consciente por parte del Estado para desarrollar las cualidades de las personas y grupos que tienen diferencias culturales, con la intención de consolidar el respeto y la creatividad, más allá de 
actitudes individuales y colectivas que mantienen el desprecio, el etnocentrismo, la explotación económica y la desigualdad social.

La interculturalidad no es un proceso de tolerancia mutua, sino que es la posibilidad de construir puentes de comunicación con el soporte de organismos que garanticen la diversidad cultural y la relación creativa. Ecuador debe ser modelo para hacer realidad esta situación.

\section{Método comparativo para la obtención de resultados científicos}

Se realiza un proceso de comparación sistemática de casos de análisis sobre la ética decolonial y su relación con el Estado de derecho con la finalidad de dilucidar la hipótesis principal: la Constitución de la República del Ecuador le da la importancia necesaria para resaltar la interculturalidad y plurinacionalidad tomando en consideración la ética decolonial.

Como expresa Collier (1993), el método comparativo se ha venido afianzando en el campo de la investigación científica en los últimos 50 años, debido a la estrecha relación que tiene con los estudios experimentales en los que los investigadores se apoyan para analizar los datos obtenidos.

Al respecto, Ramos (2012) destaca que el método comparativo es un procedimiento muy utilizado entre los métodos científicos. Junto con el método experimental y el estadístico, el método comparativo es un recurso utilizado en las ciencias sociales inherente a la investigación científica.

Este método comparativo, como cualquier método científico, se compone de tres etapas (Pliscoff y Monje, 2003). La primera etapa se estructura desde el punto de vista teórico apoyada en la elaboración de hipótesis, la cual debe centrarse en trabajos anteriores sobre la investigación. Mientras más estructuradas y claras sean las teorías, mejores serán las posibilidades de aprovechar las hipótesis relevantes para el estudio. Esto es lo que Sartori (1984) denomina conceptos empíricos (denotación) y conceptos de intensión (connotación).

La segunda etapa define criterios sobre entendidos para la obtención de la muestra, es decir, elige cómo se analiza el objeto de estudio. En este sentido, la misma debe estar identificada cuidando que los casos resulten efectivamente comparables y relevantes.

Al respecto, Nohlen (2003) menciona cuatro aspectos interesantes: el ámbito del objeto, el contexto, el tiempo y el espacio.

Y, finalmente, la tercera etapa, donde se realiza el análisis de los casos; fundamentalmente se comparan con las variables para determinar la estrategia a seguir, así como las diferencias o las semejanzas de los conceptos o teorías.

\section{Análisis del método comparativo}

De este contexto se desprenden diversas hipótesis que servirán de base a los dos estudios comparativos de la ética decolonial y su relación con el Estado de derecho en cuanto a la interculturalidad y la plurinacionalidad en el Ecuador.

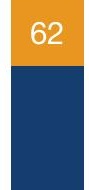


Hipótesis principal. La Constitución de la República del Ecuador le da la importancia necesaria para resaltar la interculturalidad y plurinacionalidad tomando en consideración la ética decolonial.

H1. La Constitución es factor determinante para la identificación de los derechos interculturales y plurinacionales.

H2. Cuestionar el conocimiento de la ética decolonial hacia el proceso pluriculturalista que ofrece el Estado ecuatoriano, con la intención de regir una serie de principios éticos que proporcionan respecto, seguridad e igualdad entre los habitantes.

En este contexto, se define la interculturalidad y la plurinacionalidad como las variables dependientes, mientras que las políticas, los programas, la ética y la igualdad serán las variables independientes. A partir de un análisis comparativo explicativo se podrá determinar el grado que tiene la ética decolonial y su relación con el estado ecuatoriano en cuanto a la interculturalidad y la plurinacionalidad.

Previamente y con el propósito de enriquecer la investigación, se procede a comparar y analizar la situación específica respecto a la importancia que el Estado ecuatoriano le da a la interculturalidad y plurinacionalidad teóricamente desde el ámbito constitucional, para luego interpretarlas mediante las hipótesis planteadas anteriormente.

En las últimas décadas son muchos los debates presentados acerca de la interculturalidad y el plurinacionalismo. Sin embargo, y a pesar de ser hoy en día un mandato constitucional en el Ecuador, quedan todavía algunas interrogantes que discutir debido a las diferencias marcadas entre lo que está escrito y lo que realmente está sucediendo en el territorio nacional.

La diversidad o eco-cultura en el Ecuador es la mayor riqueza actualmente; pero, al mismo tiempo, constituye un gran peligro si no se toman los correctivos necesarios, aunque sea para hacer cumplir un mandato constitucional. Por ende, es importante destacar y enfrentar que el país es rico en diversidad cultural, y que su proyección debe ser hacia todas las razas étnicas que forman ese patrimonio cultural y plurinacional en el país.

Los avisos publicitarios que hacían referencia al turismo ecuatoriano prácticamente no coincidían con lo que los turistas veían solo con hacer una pequeña caminata alrededor de la ciudad. La publicidad se direccionaba hacia personas ecuatorianas con rasgos europeos, siendo la realidad totalmente distinta y contradictoria a los hechos que se querían proyectar.

La realidad en Ecuador era su diversidad cultural. Existen mestizos, indígenas, negros y grupos de otras procedencias étnicas afroamericanas. A pesar de darle un vuelco total a este tipo de posturas, gracias a la diversidad intercultural y plurinacional reconocida constitucionalmente, todavía existen ciertos focos que quieren hacerse ver con una imagen de un país de blancos o blanco-mestizos.

La diversidad humana del Ecuador se da gracias a la variedad del clima, la geografía y las realidades ambientales. Esta pudiera ser la base de la formación de identidades regionales 
muy arraigadas. Las realidades geográficas y poblacionales se han consolidado a lo largo de la historia ecuatoriana tomando en consideración perfiles culturales y políticos. También hay cierta inclinación para tener un Ecuador homogéneo y firme en sus creencias religiosas. Por tiempo prolongado, Ecuador se inclinaba a la religión católica como parte de la razón del Estado, incluso, constitucionalmente les exigían a sus pobladores ser católicos para ser ciudadanos.

Pero el advenimiento del Estado laico permitió la libertad de conciencia y el derecho a la diversidad religiosa. Ahora, en ejercicio de ese derecho, las personas en el país practican su religión sin limitaciones legales.

Existen ciertas controversias que son evidentes cuando se comparan las realidades en cuanto al plano intercultural y plurinacional y la realidad constitucional en el Ecuador, lo que evidencia que la uniformidad de la visión que se debe dar no es verdadera. El Ecuador es definitivamente heterogéneo en todos los ámbitos. Es una entidad social y política muy compleja, en medio de una gran diversidad geográfica y cultural.

La población ecuatoriana tiene costumbres, lenguas e identidades diversas que se asentaron durante la conquista española y su continuidad republicana. A esta diversidad contribuyó, además, la implantación de comunidades afroamericanas que lograron mantener su cultura propia. También se ha levantado una tendencia a la reivindicación de los valores regionales y se han generado demandas de autonomía. Por otra parte, se han dado otras demandas de igualdad efectiva.

Algo interesante que resaltar es que los hombres se consideraban superiores a las mujeres, organizando a la sociedad y el Estado en torno a ese principio. El machismo generó grandes desigualdades y se empeñó en mantenerlas en el tiempo. Aunque las mujeres han luchado desde hace mucho por la igualdad, solo en los últimos tiempos se ha comenzado a aceptar esta dimensión de nuestra realidad y todavía hay un largo camino por recorrer. Es necesario resaltar lo importante que es hoy en día para la sociedad la posición que ocupa la mujer dentro de todos los círculos en los que se desenvuelve.

Ecuador es uno de los países de América con una tasa de juventud bastante pronunciada, pero esta situación no ha sido tomada muy en cuenta, ya que la mayoría de las situaciones son manejadas con una visión de país de adultos. Pero la verdad es que la juventud tiene grandes valores, y la mayoría de los jóvenes se preparan de manera académica, lo cual es alentador para el futuro del país. Sin embargo, sería interesante crear programas en los que se puedan mezclar la sabiduría con la nueva sangre joven para mantener actualizados los procesos en general y, al mismo tiempo, adaptarlos a las nuevas tecnologías y a la modernidad.

La heterogeneidad y la diversidad cultural se dan dentro de las realidades del subdesarrollo y en medio de un clima de pobreza. En Ecuador existen diferencias sociales de clase y en los últimos tiempos la brecha entre ricos y pobres ha aumentado. Esta diferencia de clases, en lugar de irse reduciendo, más bien se agranda, creando una brecha que se extiende a nivel internacional entre los países desarrollados y los subdesarrollados. 
Como parte del estudio comparativo en la Tabla 1 podremos ver las variables involucradas y su base de análisis, las cuales permiten enfocar la discusión y poder llegar a conclusiones concretas sobre la base de lo investigado.

Tabla 1

Variables y Base de análisis de datos

N..$^{\circ}$ Variable Base de análisis

1 Derechos interculturales y plurinacionales

Revisión y análisis del texto constitucional que avala dicho derecho

La ética decolonial hacia el proceso

2 pluriculturalista que ofrece el Estado ecuatoriano

Comparar ambos conceptos y fijar posición desde la hermenéutica

Programas y políticas como indicadores

3 sobre el Estado plurinacional e intercultural con la realidad

Revisión de lo existente y comparación con la realidad

Significado de la ética decolonial y

4 compararla con lo que persigue el Estado ecuatoriano

Revisar y analizar alcances de ambas posiciones

Fuente: Elaboración propia.

Como se puede observar, el conjunto de variables propuesto ofrece un amplio espectro de aspectos que permiten medir con cierto grado de objetividad la capacidad de entender la ética decolonial y su relación con el Estado de derecho en cuanto a la interculturalidad y la plurinacionalidad en el Ecuador.

La metodología propuesta en el estudio permite generalizar las conclusiones del análisis, dando al mismo tiempo cierta validez a las hipótesis planteadas. De este análisis ya se puede ir adentrando en el proceso de análisis para entender la verdadera razón de profundizar sobre temas que tienen apoyo constitucional, pero al mismo tiempo contrastarlos con las realidades y el día a día de los pobladores.

De acuerdo a los resultados del estudio, es importante que en el Ecuador se cree una conciencia sobre la urgencia de impulsar la interculturalidad y el plurinacionalismo. Para ello, el Estado no solo debe hacer valer las leyes existentes, sino que además debe procurar que todas sus instituciones lo apoyen de manera correcta y verdadera. El sistema educativo sería un factor importante que permitiría consolidar dichos sistemas. Hay que tener en cuenta que Ecuador es un país cuyo objetivo primordial se centra en lo intercultural y plurinacional.

Sin embargo, el mayor peligro y el más grande desafío para el Estado y la sociedad es que ni siquiera se ha reconocido la necesidad de volver intercultural el sistema educativo regular en el 
que está la inmensa mayoría de la población. Es una realidad que se debe tomar en consideración para reforzar la legitimidad de las diferencias culturales y el reconocimiento de las diversidades, así como para la promoción de la igualdad y la justicia como sus elementos fundamentales.

Si la interculturalidad y la plurinacionalidad son para las minorías, no se habrá avanzado mucho. Lo importante es que todos asuman el compromiso de levantar una sociedad apegada a lo que expresa la Constitución, y que se conozcan las diferencias desde adentro hacia afuera al mismo tiempo que se reconocen los valores, derechos y modos de vida existentes en los deberes y derechos nacionales.

No se trata de tomar medidas de Estado, ni de emitir una ley reguladora, se trata de impulsar entre todos los actores (Estado y sociedad) para lograr reconocer las diferencias y superar los prejuicios, para valorizar al otro, para construir un espacio donde se pueda regenerar la identidad intercultural y plurinacional de todos los ecuatorianos.

\section{Conclusiones}

Los derechos y la justicia social, así como la ética decolonial y la diversidad cultural, son conceptos que no deben ser negociables dentro de un Estado de derecho con el fin de privilegiar a un grupo de personas que piensan que son diferentes por el simple hecho de actuar y crecer de forma distinta ante las oportunidades de otros seres humanos. De aquí que sea tan importante que estos derechos e igualdades humanas se encuentren respaldados por normativas o leyes y, más aún, es importante velar para que los responsables de cumplirlas lo hagan de manera eficiente, en pro del bienestar de todos los ciudadanos que necesiten de ellas para la obtención y desarrollo de su crecimiento personal.

La educación juega un papel importante para desarrollar un Estado intercultural y plurinacional. Y en este sentido algunas universidades expresan su preocupación en cuanto a la desigualdad y la justicia como temas esenciales y pendientes por atacar. Su punto de vista se engloba bajo tres ejes fundamentales: en primer lugar, lo interno, el cual va referido directamente a la estructura y la visión de las universidades; en segundo lugar, la interpelación de todas las responsabilidades sociales de las instituciones superiores, tomando en consideración los nexos existentes entre la sociedad y la universidad, y por último lo relacionado con el Estado y todas las políticas educativas existentes que soportan el derecho a la educación y la igualdad entre los seres humanos, sin distinción de clase ni condición.

Por lo tanto, cuando se habla sobre la ética decolonial, esta se caracteriza por su interés de lograr la integridad y la igualdad social, pero de manera colectiva, y tomando en consideración factores sociales como la miseria que sufre gran parte de la humanidad, aunque con diferentes puntos de vistas locales, para poder afrontar de manera objetiva la igualdad de condiciones de los seres humanos. Lo importante y resaltante en este punto es que se pueda alcanzar de manera equitativa el reconocimiento y la aceptación de cada uno de los actores y que perdure el sentido común humanitario.

Lo que se rescata de todo esto es el verdadero valor humanístico que merece una población como 
la ecuatoriana, en cuanto a sus derechos como seres sociales con igualdad de oportunidades en todos los ámbitos. Así mismo, se ve la importancia de colocar al ser humano frente a sus realidades, pero dándole al mismo tiempo herramientas de todo tipo (políticas, educativas, sociales, familiares), en igualdad de condiciones y de acuerdo con los entornos que lo rodean.

En cuanto a la plurinacionalidad, representa un tema interesante de analizar debido a lo que significa dentro del proceso de evolución de los seres humanos. Existe la posibilidad de confundir términos entre plurinacionalidad e interculturalidad, teniendo cada uno un significado protagónico, durante y después del proceso de integración. Existen autores como Salazar (2016) que abordan el punto de la plurinacionalidad bajo la premisa de naciones multiétnicas, formadas por etnias diferenciadas que por un motivo $u$ otro se han unido o se han visto obligadas a vivir juntas y que juntas han conformado una historia común y comparten una memoria política.

Por su parte, De Sousa (2010) plantea la plurinacionalidad como el reconocimiento del poder convivir, tomando en consideración un solo concepto de nación de razas. Expone dos clases de naciones (la cívica y la étnica) y no por esta razón deben existir conflictos ni disputas de intereses entre ellos. De una manera coordinada e inteligente, la nación cívica puede convivir y aceptar a las naciones étnicas y ocupar los mismos espacios geopolíticos.

Tal es el caso en Ecuador con las etnias Quichua o Shuar, sin descuidar que dichas etnias no necesariamente crean un conflicto con respecto a la cívica. Es más, en el Ecuador estas etnias indígenas se descentralizan, de tal forma que buscan obtener cierto grado de autonomía política y administrativa sobre sus territorios ancestrales, pero nunca se ha planteado la independencia como alternativa.

Finalmente, teorías como la de Arriarán, (2008), Beuchot (2009), Burbano (1994), Morin (2005) y Yánez (1995) soportan las diferentes posiciones pero con fines similares a la resolución de una propuesta que pueda solventar la problemática planteada con el fin de avanzar desde los puntos de vista políticos, sociales, educativos, éticos, cultural y plurinacional, y lograr la integración definitiva de sus razas para mantener y sostener en el tiempo los derechos y obligaciones constitucionales que, en este caso, el Estado ecuatoriano ofrece a todos su pobladores. 


\section{Referencias bibliográficas}

Acosta, A. (2010). Plurinacionalidad. Democracia en la diversidad. Universidad Bolivariana, 26(1), 186-208.

Acosta, A. y Martínez, E. (2011). La naturaleza con derechos: de la filosofía a la política. Quito, Ecuador: Ediciones Abya-Yala.

Alavez, A. (2014). Interculturalidad: Conceptos, alcances y derecho. México: GPPRD.

Almeida, I. (10 de enero de 2018). Ensayo: Interculturalidad en Ecuador, una realidad incierta [Mensaje en un blog]. Recuperado de https://lalineadefuego.info/2018/01/16/ ensayo-interculturalidad-en-ecuador-una-realidad-incierta-por-ileana-almeida/

Arce, R. (2017). Interculturalidad en perspectiva de complejidad. Servindi. Recuperado de https://www.servindi. org/ actualidad-opinion/19/12/2017/interculturalidad-en-perspectiva-de-complejidad.

Arriarán, S. (2008). La filosofía latinoamericana en el siglo XXI, después de la postmodernidad. México: Pomares.

Beuchot, M. (2009). Hermenéutica analógica y educación multicultural. México: Plaza y Valdés

Burbano, B. (1994). La educación como identificación cultural. Quito, Ecuador: Abya-Yala.

Céspedes, E. (2012). Filosofía latinoamericana en la época de la globalización: colonialidad, interculturalidad y filosofía de la liberación. Praxis, 66(1), 173-180.

Collier, D. (1993). The comparative method. American Political Science Association, 6(1), 105-119.

Constitución de la República del Ecuador (2008). Decreto Legislativo 0. Registro Oficial 449 del 20 de octubre de 2008.

Cuji, L. (2012). Una paradoja de la interculturalidad como descolonización de la educación superior. Diversidad, 10(1), 40-53.

De Sousa, B. (2010). Refundación del Estado en América Latina. Perspectivas desde una epistemología del Sur. Perú: Instituto Internacional de Derecho y Sociedad.

Fernández, F. (2012). Una experiencia en educación intercultural. Talleres en la escuela Taigoché, Rosario, Argentina. Diversidad, 10(1), 70-78.

Fonseca, M. y Jerrems, A. (2012). Pensamiento decolonial: ¿Una nueva apuesta en las relaciones internacionales? Relaciones Internacionales, 19(1), 103-121. 
Kant, I. (1996). Fundamentación de la metafísica de las costumbres. Barcelona, España: Ariel.

Maldonado-Torres, N. (2008). La descolonización y el giro descolonial. Revista Tábula Rasa, 9(1), 61-72.

Morin, E. (2005). Los siete saberes necesarios para la educación del futuro. Quito, Ecuador: Santillana.

Nohlen, D. (14 de diciembre 2013). El Método comparativo [Mensaje en un blog]. Recuperado de www.rzuser. uni-heidel berg.de

Núñez, D. M. y González, C. (2014). Interculturalidad como horizonte y posibilidad de relaciones hermanas. Recuperado de http://biblioteca.clacso.edu.ar/Republica_Dominicana/ccp/20170217044144/pdf_698.pdf.

Paz, M. (25 de agosto de 2015). ¿Qué significa ser un Estado Plurinacional? [Mensaje en un blog]. Recuperado de https://www.la tercera.com/noticia/que-significa-ser-un estado-plurinacional/

Pliscoff, C. y Monje, P. (2003). Método comparado: un aporte a la investigación en gestión pública. XIII Congreso Internacional del CLAD sobre la Reforma del Estado y de la Administración Pública, 1-13. Recuperado de http://unpan1.un.org/intradoc/groups/public/documents/CLAD/clad0047327.pdf

Polo, M. (2016). Decolonialidad, interculturalidad y reconocimiento. Vox Juris, 32(2), 65-70.

Ramos, L. (2012). Método comparado: precisiones y características. Revista de Ciencia Política, 16(1), 1-28.

Salazar, D. (2016). ¿Cómo definir la interculturalidad? Enfoque, 30(1), 1-4.

Sartori, G. (1984). La política: lógica y método de las ciencias sociales. México: FCE.

Soazo, C. (2017). Fundamentos para una aesthesis cultural decolonial. Opción, 33(83), 24-41.

Taylor, C. (1993). Propósitos cruzados: El debate liberal-comunitario. Buenos Aires, Argentina: Paidós.

Tirzo, J. y Hernández, J. G. (2010). Relaciones interculturales, interculturalidad y multiculturalismo; teorías, conceptos, actores y referencias. Cuicuilco, 17(48), 11-34.

UNESCO (2005). La Convención sobre la protección y promoción de la diversidad de las expresiones culturales. París, Francia: UNESCO.

Villoro, L. (2000). Sobre el principio de la injusticia: La exclusión (Octavas Conferencias Aranguren, 1999). Isegoría Revista de Filosofía Moral y Política, 22(1), 103-142.

Vitória, P. (2017). Hacia una propuesta pluriversal, crítica y decolonial sobre democracia y derechos humanos (tesis doctoral). Universidad Pablo Olavide, Servilla, España. 
Walsh, C. (2013). Pedagogías decoloniales: prácticas insurgentes de resistir, (re)existir y (re)vivir. Quito, Ecuador: Abya-Yala.

Walzer, M. (2001). Just and unjust wars: a moral argument with historical illustrations. Barcelona, España: Paidós.

Yánez, C. (1995). La educación indígena en el Ecuador. Quito, Ecuador: Instituto de capacitación Municipal. 